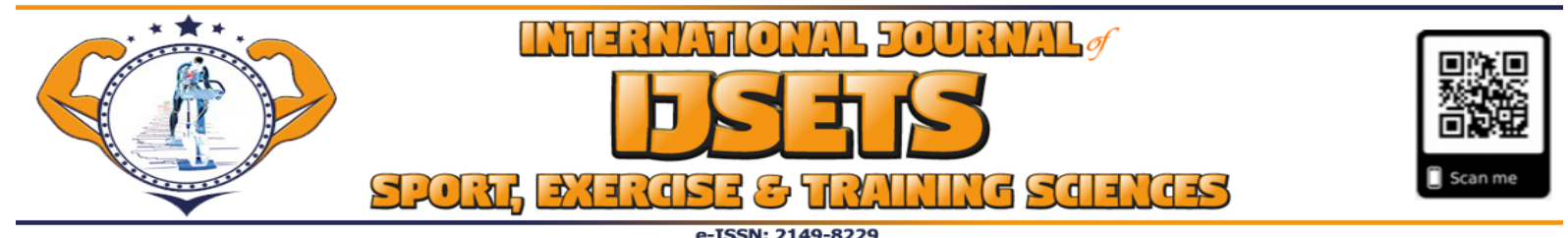

\title{
Effect of Dehydration on The Development of Endurance and Vertical Elasticity in High-level Basketball Players
}

\section{Mohamed Salah Eddine BENSETTI ${ }^{1}$, Kada BELKEBICHE ${ }^{2}$, Abdellah MERZOUK ${ }^{3}$}

\begin{tabular}{|c|c|}
\hline Abstract & Keywords \\
\hline $\begin{array}{l}\text { Aim: The objective of this study is to observe and verify the effect of dehydration about the } \\
\text { performance of physical abilities of endurance and vertical elasticity among high-level basketball } \\
\text { players and compare them to the various studies that have been carried out so far and provide } \\
\text { adequate recommendations for a dual objective, to achieve performance and preserve the athlete's } \\
\text { health. }\end{array}$ & $\begin{array}{r}\text { Hypohydration, } \\
\text { Fluid Balance, } \\
\text { Aerobie Performance, } \\
\text { Explosive Force }\end{array}$ \\
\hline $\begin{array}{l}\text { thods: This study followed a quantitative descriptive research design. We performed tests and } \\
\text { tests of vertical elasticity through the Squat Jump (SJ) test for the same group being normally } \\
\text { lrated and being dehydrated (on an empty stomach). Then, we followed up with the } 1 / 2 \text { cooper } \\
\text { s and re-tests for endurance evaluation for the same group being normally hydrated and being } \\
\text { lydrated (fasting). Comparison between the different tests during the three periods of the } \\
\text { son. }\end{array}$ & \\
\hline $\begin{array}{l}\text { For the first set of results related to the Squat Jump throughout the three periods, we } \\
d \text { a slight difference but statistically significant between the tests for the two states of } \\
\text { on. The second sets of results related to the half Cooper were even more confirming and } \\
\text { ng the first set of results. During the three We recorded statistically significant difference } \\
\text { states of hydrations, with P value }<0.001 \text {. }\end{array}$ & $\begin{array}{r}\quad \underline{\text { Artic }} \\
\text { Received:14.1 } \\
\text { Accepted:09.1 }\end{array}$ \\
\hline $\begin{array}{l}\text { Conclusion: Through this study, we observed the effect of dehydration on specific physical } \\
\text { abilities in our basketball players. It has been found that dehydration negatively affects the } \\
\text { development of their physical abilities. This leads to the implementation of the individualization } \\
\text { of hydration strategies. }\end{array}$ & DOI:10.18826/useeabd.840312 \\
\hline
\end{tabular}

\section{INTRODUCTION}

To know the harmful effects of dehydration on athletic performance, it will be necessary to assess the effect of exercise and training sessions on the athlete's water status. According to Guezzenec, (2011), sweating could increase considerably during physical exertion to reach $4 \mathrm{l} / \mathrm{h}$ in extreme conditions. Thus, if the fluid intake does not compensate for the fluid losses, a state of dehydration appears and a decline in physical and mental performance could be observed (Sawka et al., 2007).

Knowing that high-level athletes devote a lot of time and effort to perfecting their technical movements, their physical conditions, and their tactical preparation, they must not neglect the nutritional aspect, in particular hydration and their management of the water balance which is part of their recovery and which directly influences their performance. In this context, sometimes coaches, athletes, physical trainers, are faced with incomprehensible sporting failure, it could be that poor management of the water balance could justify this failure; it can be a cause of poor performance.

Because of the significant change in the intensity and frequency of basketball playing actions, with an increase in the duration of efforts and a reduction in break times, today the sessions must be more demanding, in particular, in physical engagement, because athletes must be able to withstand harder and more extensive workloads from an athletic preparation standpoint. This intermittent aspect of basketball was further accentuated by the change in the discipline's regulations in 2001, namely the rule of 24 and 8 seconds, 4 periods of play of 10 minutes and 5 time-outs per team.

In team sports, it is recognized that the athlete has limited possibilities to hydrate. In this case, the athlete should take advantage of stoppage time to rehydrate while planning the volume to ingest on each occasion (Maughan and Shirreffs, 2010). This is the case with basketball where he has the opportunity

The role and contributions of each authors as in the section of IJSETS Writing Rules "Criteria for Authorship" is reported that: 1. Author: Contributions to the conception or design of the paper, data collection, writing of the paper and final approval of the version to be published paper; 2. Author: Data collection, preparation of the paper according to rules of the journal, Statistical analysis, interpretation of the data and final approval of the version to be published paper; $\mathbf{3}$. Author: Contributions to the conception or design of the paper, data collection, Statistical analysis,

${ }^{1}$ Corresponding Author: Laboratoire d'Optimisation des Programme d'Activité Physique et Sportive LABOPAPS. STAPS universitéIbn Badis Mostaganem, Algeria, bensettis@yahoo.fr. ORCID ID: https://orcid.org/0000-0001-8886-5217

${ }^{2}$ Laboratoire d'Optimisation des Programme d'Activité Physique et Sportive LABOPAPS. STAPS université Ibn Badis Mostaganem, Algeria

${ }^{3}$ Laboratoire d'Adaptation Physiologique à l'Exercice et Réadaptation à l'Effort APERE STAPS, université Jules Vernes Amiens, France 
to hydrate during the 5 timeouts for each team, therefore 10 breaks during a match which can be up to 81 minutes depending (Travaillant et al., 2003).

Dehydration for basketball players is very important according to (Broad et al., 1996). It is located between $1.4 \mathrm{l} / \mathrm{h}$ and $1.6 \mathrm{l} / \mathrm{h}$, which requires serious management by the adoption of well-defined strategies to compensate for it in training as in competition, especially with us in Algeria., where generally the climate is mild, rather warm ( 8 months $/ 12)$.

The purpose of this article is to answer the main research question, namely whether dehydration has an impact on the development of the endurance and vertical elasticity of basketball players. Therefore, we aim to show the importance of good hydration and convey a clear message of putting in place strategies and hydric plans for the dual goal of achieving performance and preserving our athletes' health.

\section{METHOD}

\section{Participants}

Study sample: Our study was carried out on the senior male team of an Algerian National Club from the first league which consists of 20 players with the following characteristics:

Table 1: Characteristics of the sample GSP

\begin{tabular}{cc}
\hline Age (years) & $23 \pm 0,62$ \\
\hline Weight $(\mathrm{kg})$ & $80.21 \pm 8.80$ \\
\hline Height $(\mathrm{cm})$ & $189 \pm 6.46$ \\
\hline
\end{tabular}

Research Protocol: Our study protocol consists of evaluating and comparing the strength and special endurance of the high-level basketball player about two different states, normally hydrated and dehydrated, during three different periods of the season, therefore, tests were carried out at two days of delay, first in a normal state of hydration with the possibility of deteriorating during the test at will and second in a state of dehydration, (voluntary dehydration, restriction of deterioration 6 hours before the tests and during the test until the end of the session, (on an empty stomach).

Hypotheses: We suppose that through the physical tests carried out on a group of high-level athletes during the 3 main phases of the season (preparatory, competitive and transient) indicate to us that dehydration negatively affects the process of development of endurance capacities as well as the vertical elasticity capacities of the basketball player.

\section{Data collection}

Protocol 1. Squat Jump (SJ): Using a Squat Jump to measure non-plyometric dry elasticity, without stretching. We did a 15-minute warm-up, plus a few sets of sheathing movements followed by a 3minute break before each test. We measure the height of the jump of each athlete "the vertical elasticity" and three repetitions were performed looking for the maximum height.

Protocol 2. The Half Cooper or the 6 Minute Test: The Half Cooper test was used to calculate the Maximal Aerobic Speed (MAS) of each athlete; it indicates the speed which is witness to the capacity of each athlete to oxygenate his muscles during the effort, it is essential to establish the intensities of the effort of the program. of training. The goal is simple, it is to try to achieve or cover the greatest distance in 6 minutes, after a warm-up of 10 to 15 minutes in slow running followed by 2 fast runs of 40 to 50 meters with return throttled on the distance to cool down, then rest for 2 minutes before starting the test. The value of the MAS corresponds to the distance travelled in meters divided by one hundred (D/100).

\section{Statistical analysis}

In the analysis of the data, the arithmetic mean and standard deviation values for the measurement results of each variable were calculated separately and a table was created. The significance between the measurement values of the groups was tested with the Mann Whitney-U test.

\section{RESULTS}

Comparison between the different tests during the 3 periods of the season. For the processing of the data collected, we calculated the arithmetic mean, the standard deviation, the variance and the coefficient of 
variation. The arithmetic means the sum of the measured values divided by their number it determines the average value of a series of calculations. The question may arise if the result of the averages is different. This difference can be due to chance as it can be a significant fact.

Figure 1. Vertical elasticity results

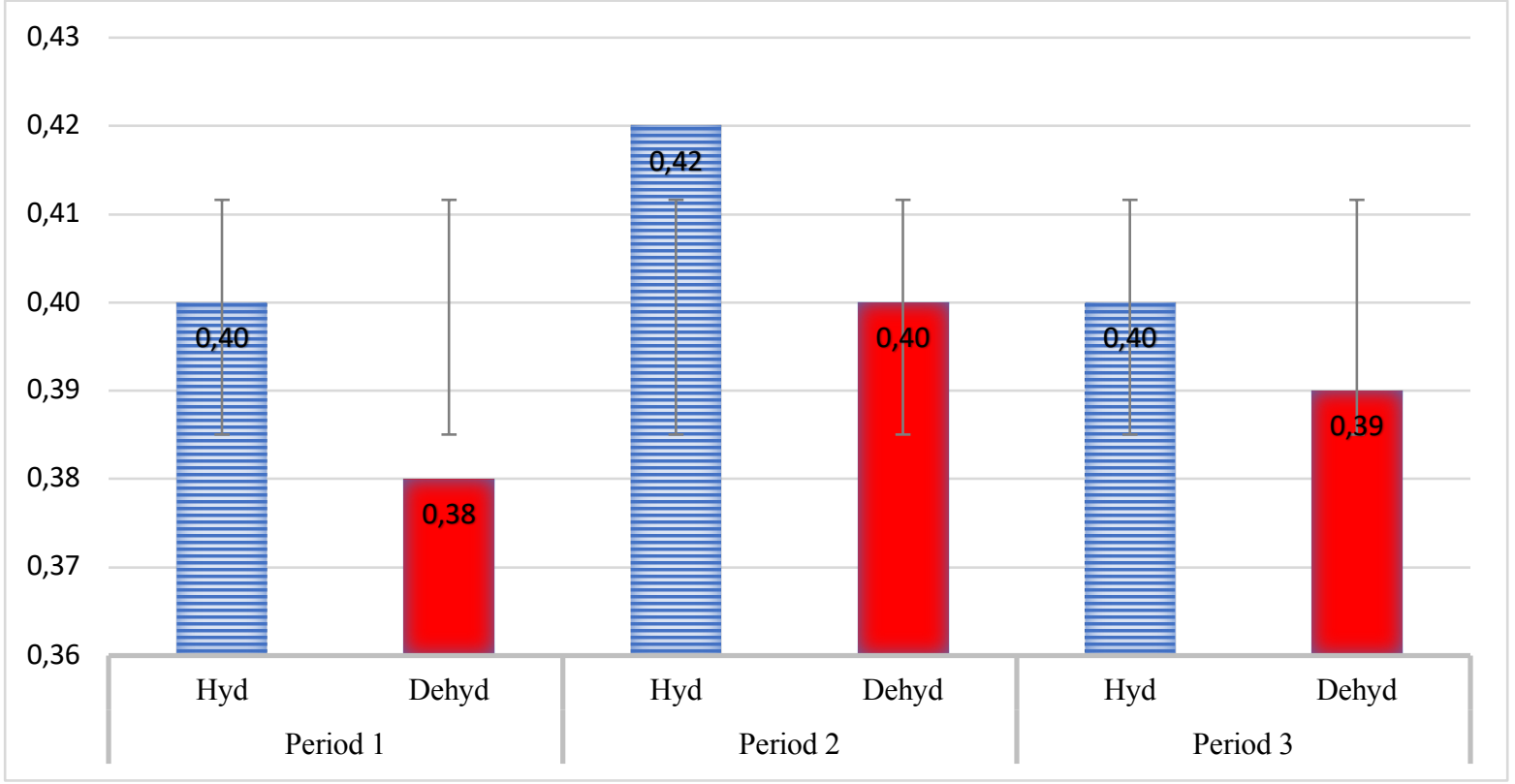

Note: Hyd: hydration condition, Dehyd: dehydrated condition-

We recorded a slight difference between the tests for the 2 states of hydration, however, we recorded an average of $0.40 \mathrm{~cm}$ being normally hydrated and $0.38 \mathrm{~cm}$ being dehydrated in period 1 , an average of $0.42 \mathrm{~cm}$ being normally hydrated and $0.40 \mathrm{~cm}$ being dehydrated in period 2 and an average of $0.40 \mathrm{~cm}$ being normally hydrated and $0.39 \mathrm{~cm}$ being dehydrated in period 3 . All differences are very significant $(\mathrm{p}<0.001 * * *)$.

Figure 2. Vertical elasticity changes between periods

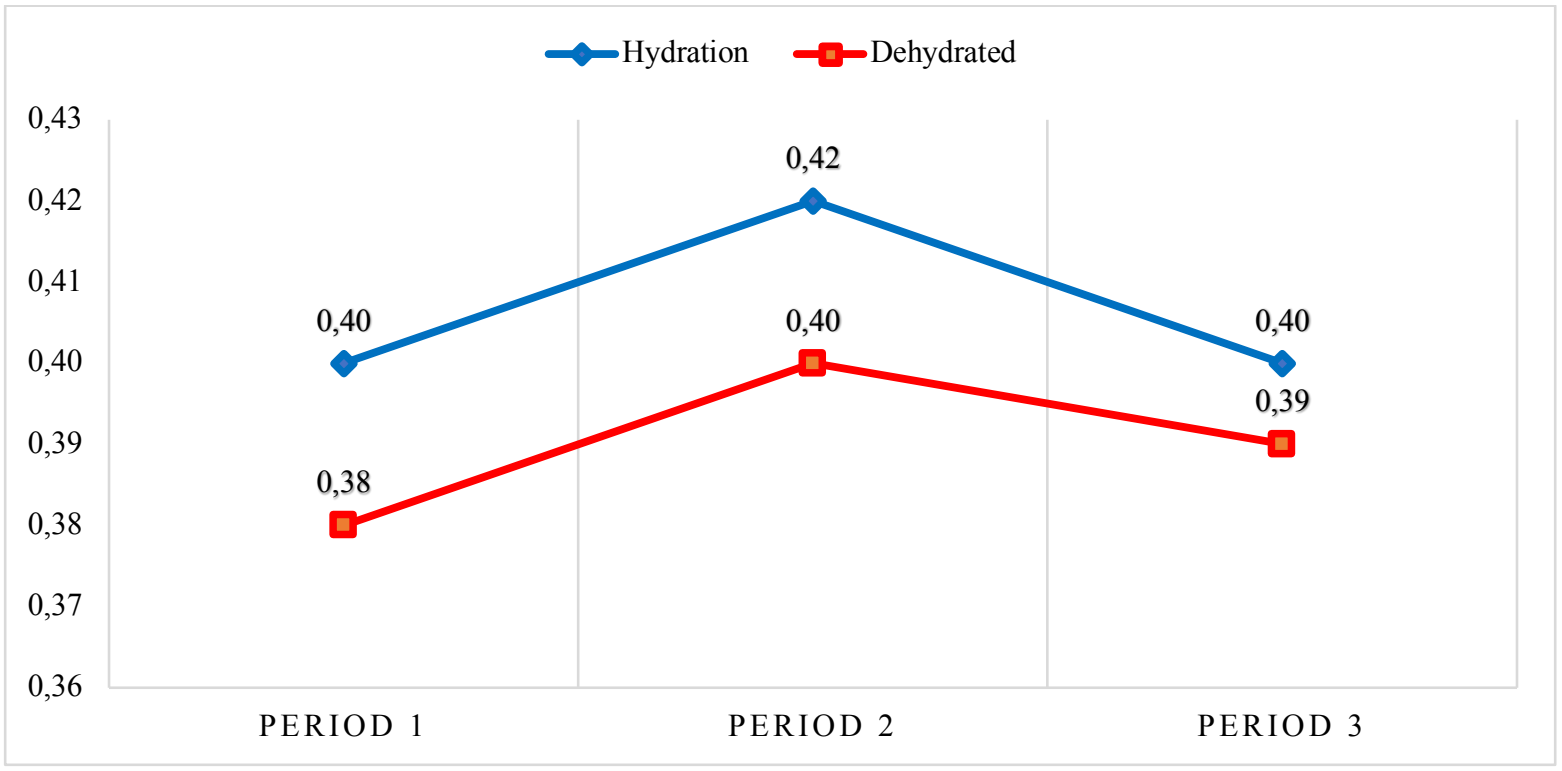

As a result of the vertical elasticity measurements, the averages of the hydration and dehydrated groups were evaluated. The change in averages between periods is revealed. It was observed that there was a change in the averages. 
Figure 3. Endurance results

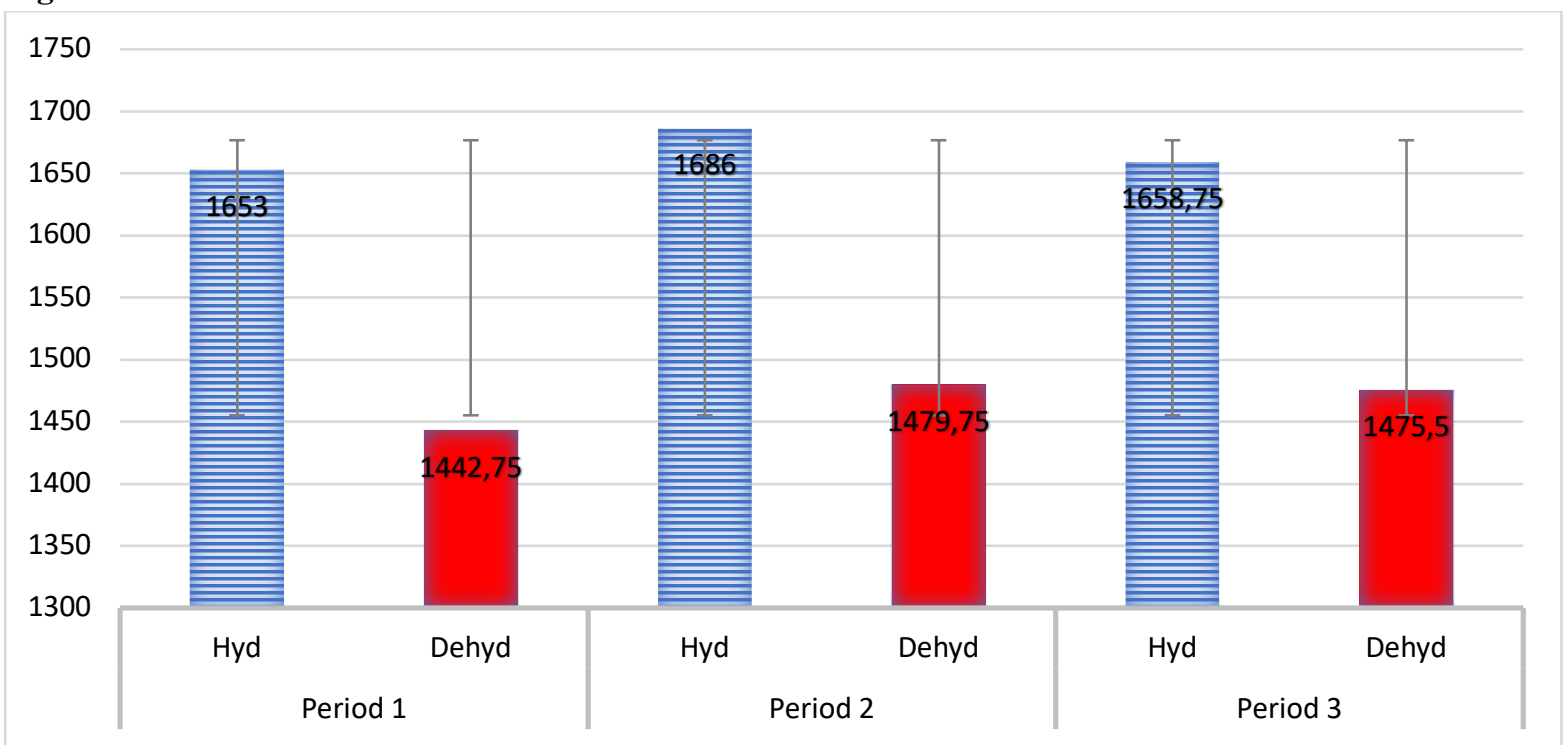

Note: Hyd: hydration condition, Dehyd: dehydrated condition

We recorded a difference between the results of the half cooper test ( 6 minutes) for the two states of hydration during the 1st period because we recorded an average of $1653 \mathrm{~m}$, being normally hydrated and 1442.75 being dehydrated. During period two, we also recorded an average of $1686 \mathrm{~m}$, being normally hydrated and $1479.75 \mathrm{~m}$ being dehydrated. Moreover, period three noted an average of 1658.75 $\mathrm{m}$, being normally hydrated and $1475.5 \mathrm{~m}$ being dehydrated. All differences are very significant $(\mathrm{p}$ $<0.001 * * *)$.

Figure 4. Endurance changes between periods

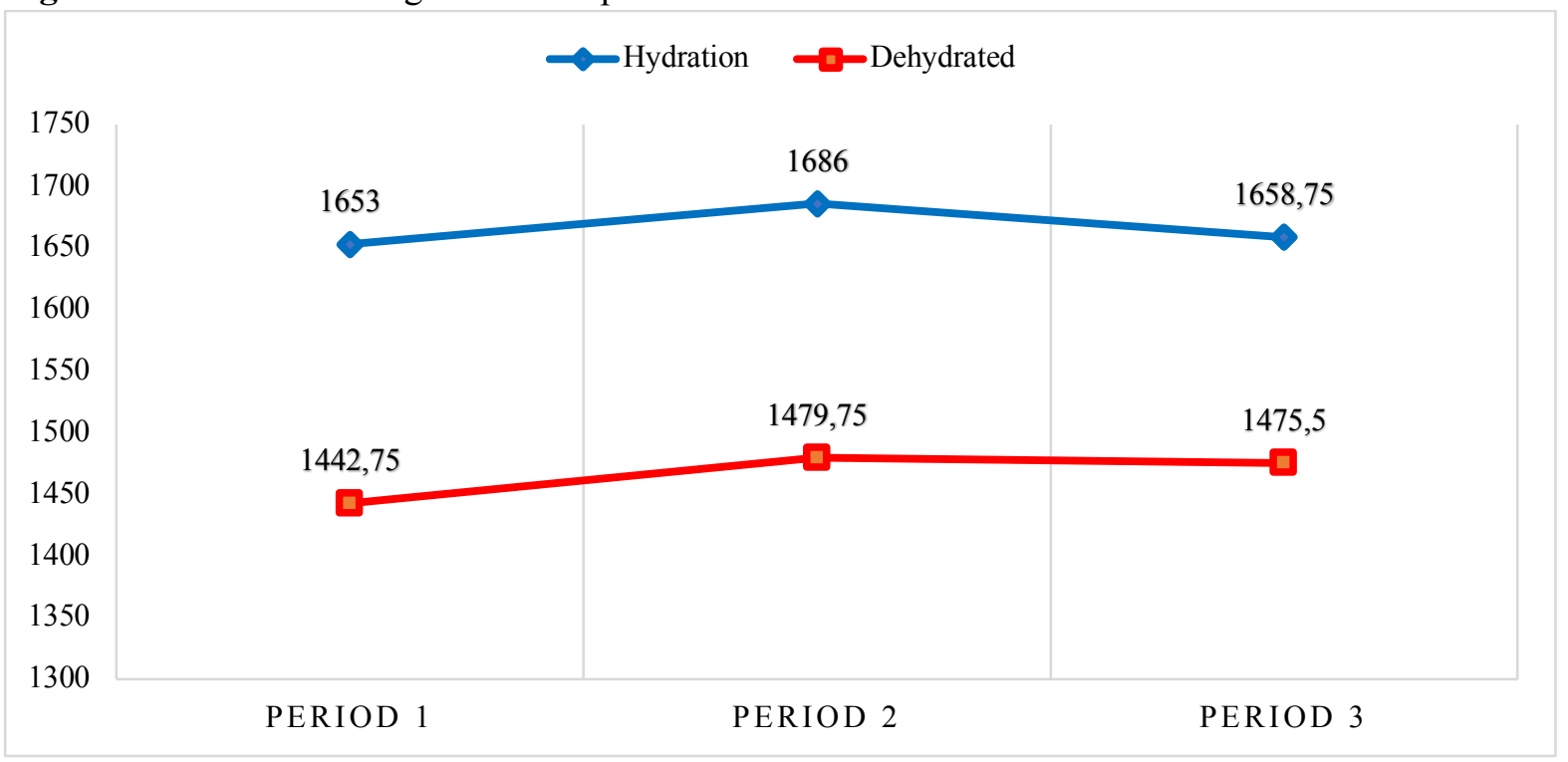

As a result of the endurance measurements, the averages of the hydration and dehydrated groups were evaluated. The change in averages between periods is revealed. It was observed that there was a change in the averages.

\section{DISCUSSION}

The findings of the data analysis relate the water losses and the performance of endurance capacity and strength in high-level Algerian basketball players allowing us to conduct a comparison of the present 
study with other related works. Hence, the comparison will assess the place given to hydration and its effect on the development of the physical abilities specific to basketball.

The majority of authors and researchers indicate that dehydration has a negative influence on physical and intellectual performance. In this context, (Hawley et al., 1994) indicate that after $2 \%$ of water loss from our body mass, our body is only functioning at $80 \%$ of its capacity. Data from the literature generally shows that dehydration greater than or equal to $2 \%$ is detrimental to performance; however current recommendations state to drink enough fluids to limit dehydration of $\geq 2 \%$. Otherwise, performance will be adversely affected. (Sawka et al., 2007; Shirreffs and Sawka, 2011).

We were therefore interested during our study to observe the effect of dehydration on different abilities first of all the elasticity which is very important in the basketball player, an essential quality in the game since we analyse the game. We found that During a basketball game, players average 100 jumps, which is a significant number and requires adequate athletic preparation.

The results of the squat jump test revealed a very significant difference between the two states of hydration with a decrease in strength performance. It is believed that the basketball player is far from having all his physical faculties, muscular and nervous when he is dehydrated. The dehydration state doesn't allow it to perform in the same way as being normally hydrated.

According to our study, dehydration has only $1.37 \%$ of the body mass. It has negatively affected the particularity of elasticity in our basketball players. Although recent research widely agrees on the decrease in performance starting at $2 \%$ of fluid loss (dehydration). Hence, this confirms our hypothesis but goes against previous studies.

For the Half Cooper test where we wanted to see the effect of dehydration on the endurance faculties of our basketball players, the results showed us a very significant difference during the 3 periods and this confirms our hypothesis knowing that we are at $1.37 \%$ dehydration only. However, Professor Goulet from Sherbrook University (Canada) considers that mild dehydration does not affect endurance performance, he has shown during a meta-analysis that during a protocol simulating the performance of the type "Against the Clock", dehydration levels of up to $4.3 \%$ CP do not adversely affect performance and drinking when thirsty would optimize performance in endurance athletes (Goulet's tests were performed on a bicycle) (Goulet, 2013). This discrepancy is due to the type of sport and activity. The subjects of the study were cyclists, and our subjects are basketball players which could affect the final results. These works are generally applied to marathon runners or cyclists. Aside from basketball, which has its specificities and its intermittent nature, it seems logical to us that the results will be different.

According to (Goulet, 2013), the impact of slight dehydration on endurance performance is presented by differentiating between two types of protocols: a) Protocol approaching the real race conditions such as "against the clock"; and b)- Further away from natural competition conditions maintaining a precise intensity as long as possible.

In both protocols, endurance performance was compared in subjects who were properly hydrated and subjects who were dehydrated by exertion. The results of this analysis show that under conditions close to reality, dehydration following exercise improves endurance performance but not significantly $(+0.09 \%, \mathrm{P}=0.9)$ whereas, in the event of protocol further from reality, dehydration significantly reduces performance $(1.91 \% \mathrm{P} / 0.05)$ (Goulet, 2013).

Our findings comply with certain Goulet (2013) studies to some extent. In other words, the main finding of our study reveals that the dehydration negatively the developments of endurance confirm the results of the second protocol of Goulet (2013).

\section{CONCLUSION}

Hydration is one of the pillars of sporting success. To reach the top with high results, it should occupy a prominent place among our top athletes. However, managing and optimizing its water potential should be a major concern and a concern for all actors in the sports world, namely; athletes, coaches, doctors, physical trainers... etc.

Through this study, we observed the effect of dehydration on specific physical abilities in our basketball players. It has been found that dehydration negatively affects the development of their physical abilities as it has been mentioned sometimes through research. Knowing that the results of the tests carried out during our study are not all consistent with the data put forward by the scientific literature which agree on the idea that from dehydration of $2 \%$ that the physical capacities of an athlete are impaired. We have found that even minimal dehydration, between $1 \%$ and $2 \%$ in our basketball 
players, has a negative influence on the physical performance and especially the endurance capacities of our athletes. Contrary to what we have known from the work of Cheung et al. (2015) who stated that slight dehydration (between 2\% and 3\%) does not affect endurance performance. These works are generally applied to marathon runners or cyclists. Aside from basketball, which has its specificities and its intermittent nature, it seems logical to us that the results will be different.

In basketball, the constraints of time and pressure are decisive, especially after the change of regulations in 2001. The new regulations such as the rule of 5-time outs, the rule of 24 seconds, the rule of 8 seconds have made the competition harder and the efforts more intense.

As a recommendation, players are encouraged to drink during timeout and stoppage time optimally and intelligently. Without the mistake of drinking large amounts of water, that could provoke a gastric discomfort which inevitably induces a poor performance.

To achieve high performance with optimum results, our basketball players must adopt an adequate, personalized, and well-studied hydration strategy. This could be achieved by knowing how to quantify their sweating rates to know the volume of water or drink needed to consume during training or in competition. They must be, always, well-hydrated in competition and training as well.

\section{REFERENCES}

Broad, E. M., Burke, L. M., Cox, G. R., Heeley, P., \& Riley, M. (1996). Bodyweight changes and voluntary fluid intakes during training and competition sessions in team sports. International Journal of Sports Nutrition and Exercise Metabolism, 6(3), 307-320.

Cheung, S. S., McGarr, G. W., Mallette, M. M., Wallace, P. J., Watson, C. L., Kim, I. M., \& Greenway, M. J. (2015). Separate and combined effects of dehydration and thirst sensation on exercise performance in the heat. Scand J Med Sci Sports, 25(1985):104-11.

Goulet, E. D. (2013). Effect of exercise-induced dehydration on endurance performance: evaluating the impact of exercise protocols on outcomes using a meta-analytic procedure. British Journal of Sports Medicine, 47(11), 679-686.

Guezennec, C. Y. (2011). Les boissons de l'effort: bases physiologiques de leurs utilisations et composition. Cahiers de Nutrition et de Diététique, 46(1), H46-H53.

Hawley, J. A., Walsh, R. M., Noakes, T. D., \& Dennis, S. C. (1994). Impaired high-intensity cycling performance time at low levels of dehydration. International Journal of Sports Medicine, 15(07), 392-398.

Maughan, R. J., \& Shirreffs, S. M. (2010). Dehydration and rehydration in competitive sport. Scandinavian Journal of Medicine \& Science in Sports, 20, 40-47.

Sawka, M. N, Noakes, T. D. (2007). Does dehydration impair exercise performance?. Med Sci Sports Exerc, 39(8):1209-17.

Sawka, M. N., Burke, L. M., Eichner, E. R., Maughan, R. J., Montain, S. J., \& Stachenfeld, N. S. (2007). American College of Sports Medicine position stand. Exercise and fluid replacement. Medicine and Science in Sports and Exercise, 39(2), 377-390.

Shirreffs, S. M., \& Sawka, M. N. (2011). Fluid and electrolyte needs for training, competition, and recovery. Journal of Sports Sciences, 29(1), 39-S46.

Travaillant, G., \& Cometti, G. (2003). Analyse des efforts en basket. CEP Dijon. UFR STAPS Dijon Université de Bourgogne, 1-21.

\section{CITATION OF THIS ARTICLE}

Bensetti, M.S.E., Belkebiche, K. \& Merzouk, A. (2021). Effect of Dehydration on The Development of Endurance and Vertical Elasticity in High-level Basketball Players. International Journal of Sport, Exercise \& Training Sciences - IJSETS, 7(1), 6-11. Doi: 10.18826/useeabd.840312 